\title{
Conflictos éticos en el aprendizaje de los residentes
}

\author{
J. A. GARRIDO SANJUÁN \\ Servicio de Medicina Interna. Hospital A. Marcide-Novoa Santos. Área Sanitaria de \\ Ferrol. La Coruña
}

ETHICS CONFLICTS IN LEARNING AND TEACHING MEDICAL SPECIALITIES

Garrido Sanjuán JA. Conflictos éticos en el aprendizaje de los residentes. An Med Interna (Madrid) 2006; 23: 493-502.

\section{INTRODUCCIÓN Y FUNDAMENTACIÓN}

La dimensión moral es inherente a la práctica de las profesiones sanitarias. Una de las actividades casi inseparables de la propia actividad profesional del médico es la contribución al aprendizaje de otros médicos en las distintas fases de su formación: estudiantes de medicina, especialización médica (residentes, MIR), formación continuada de otros compañeros, etc. Es importante la preocupación por la reflexión y mejora de la moralidad de las acciones profesionales de los médicos, dando muestra de ello el aumento de la literatura que hace referencia a la ética médica o a la bioética, no sólo en las revistas especializadas en este campo, sino también en las revistas generales de difusión de conocimientos científicos médicos o las específicas de las distintas especialidades médico-quirúrgicas.

Esta preocupación es la que ha hecho desarrollarse la Bioética que tiene su origen en la aparición de conflictos éticos motivados principalmente por tres razones: los grandes avances científico-técnicos, el cambio en las relaciones sanitarias y la universalización de la asistencia. La preocupación por la dimensión ética de la investigación precedió a la de la asistencia y de ella proceden en parte los principios bioéticos que nos ayudan a tomar decisiones en la clínica.

Por tanto de las tres dimensiones que se imbrican en la actividad de nuestros centros sanitarios (asistencia, investigación y docencia) es la docencia la que ha permanecido más al margen de la preocupación por su dimensión ética. Sin embargo esta dimensión profesional también se ha visto inmersa en los cambios que hemos referido como origen de los conflictos bioéticos, y por tanto han surgido también los problemas morales. Además esta actividad se ha visto también sometida a una reflexión, no sólo para la adaptación de la formación a los rápidos cambios profesionales, sino para adaptarse a nue- vos conceptos pedagógicos. De una forma esquemática este último punto podríamos resumirlo en el paso de una enseñanza centrada en el docente a un aprendizaje centrado en el alumno (en este caso el estudiante de medicina o el residente).

La búsqueda de orientaciones sobre la ética de la formación especializada para incluir este tema en una formación reglada a los tutores de residentes en la que estoy participando, encontrando escasas reflexiones sobre la misma en nuestra literatura médica, me ha conducido a escribir este texto. Iré revisando los distintos puntos aquí introducidos con la pretensión de que sirvan de partida para la discusión y mejora de orientaciones que permitan detectar, solucionar y prevenir conflictos éticos que son inherentes, al igual que a la asistencia, a la docencia de la medicina. El texto no pretende sentar directrices sino orientar una deliberación sobre el tema que ayude a definir guías de decisión.

Aunque muchos de los puntos a revisar son probablemente extrapolables a la formación de alumnos de medicina me centraré en la formación residentes por ser el campo que conozco y trabajo. El matiz esencial que marca la diferencia es que el residente tiene ya una capacitación como médico, al menos teórica, para muchos actos generales, que no tiene el estudiante.

\section{LA ENSEÑANZA DE LA MEDICINA ¿ES INHERENTE A NUESTRA ACTIVIDAD PROFESIONAL?}

Buscando los orígenes de la respuesta a esta pregunta en la historia podemos remontarnos a revisar el texto del código hipocrático (Tabla I). El compromiso con la formación de otros médicos, con las limitaciones y restricciones marcadas por la época para mantener controlado el acceso a la profesión, con claro corporativismo, está ligado al mismo acto de 


\section{TABLA I}

JURAMENTO HIPOCRÁTICO

Juro por Apolo médico, por Asclepio, Higía y Panacea, así como por todos los dioses y diosas, poniéndolos por testigos, dar cumplimiento en la medida de mis fuerzas y de acuerdo con mi criterio al juramento y compromiso siguientes:

- Tener al que me enseñó este arte en igual estima que a mis progenitores, compartir con él mi hacienda y tomar a mi cargo sus necesidades si le hiciese falta;

- Considerar a sus hijos como hermanos míos y enseñarles este arte, si quieren aprenderlo, de forma gratuita y sin contrato o compromiso;

- Hacerme cargo de la preceptiva, la instrucción oral y demás enseñanzas de mis hijos, de los de mi maestro y de los discípulos que hayan suscrito el compromiso y estén sometidos por el juramento a la ley médica, pero de nadie más.

$-\ldots . . . .$.

compromiso necesario para empezar a ejercer la profesión. Otra argumentación para responder a la pregunta la encontramos en el mismo significado de la palabra doctor ("docere": enseñar).

En nuestro siglo XXI creo que la obligación de contribuir a formar a otros como médicos y a médicos como especialistas es inherente al ejercicio de nuestra profesión. Como dicen Stone y cols., "la identidad del médico contiene implícitamente la identidad del maestro" (1). Si esta afirmación puede dejar algún resquicio de duda o argumentación en contra de forma genérica, cuando la aplicamos a la actividad de los médicos en los centros públicos o privados acreditados para la docencia estas posibles dudas se diluyen. Desarrollar la actividad profesional en estos centros nos obliga a la participación en la enseñanza de aquellos con los que los centros y servicios se han comprometido. Será de forma diferente para cada uno, con responsabilidad y dedicaciones distintas y a demanda de los responsables de dicha formación. En el caso de los residentes, desde los tutores, máximos responsables de la formación especializada, a los médicos responsables de las rotaciones $\mathrm{u}$ otros médicos que coinciden en una "oportunidad docente" puntual con el residente. Aunque no todos participen en la evaluación final o sumativa, todos deberían participar en mayor o menor grado en la evaluación formativa. Uno de los problemas del actual sistema MIR es que no hay una adecuada conciencia, y por tanto no se plasma en su desarrollo práctico, de que lo acreditado para la docencia es la Unidad Docente y no es el jefe de servicio, el tutor o tal o cual persona dentro de la Unidad. El refuerzo y reconocimiento de la importancia que tiene cada persona de la Unidad como formador de los residentes adscritos a la misma puede estimular la satisfacción con dicha labor docente y los deseos de mejorar su participación y habilidades en esa faceta profesional (1).

Además de que defiendo que ésta es una obligación ética, e incluso en muchos casos legal y laboral, cuando trabajamos en una Unidad Docente acreditada, hay también un motivo "utilitarista" para que estemos estimulados a la participación en la docencia. De la presencia de estudiantes y residentes en nuestras Unidades nos beneficiamos todos por el estímulo que supone para nuestra formación continuada, que aparece también como obligación ética e inherente a nuestra profesión, ligada al principio de no-maleficencia (sólo podré continuar ofertando lo indicado al paciente si conozco lo indicado en ese momento concreto por estar al tanto de los avances científicotécnicos).

Esta identificación del médico con la labor de formador, docente o incluso "maestro", no es incompatible con otra de las facetas que es necesario seguir desarrollando para mejorar nuestro sistema de especialización como es la "profesionalización" de los tutores. Por otro lado tampoco supone un argumento en contra de la necesidad de formar en métodos pedagógicos y en técnicas y herramientas docentes al menos a parte de los componentes de la Unidad Docente. Una cosa es la necesidad y obligación de contribuir a la formación de los alumnos y residentes asignados a la Unidad y otra que esta capacidad sea innata y no claramente susceptible de aprendizaje y mejora. Hay evidencias de que las características demandadas en los docentes apreciados como modelos a seguir son susceptibles de aprendizaje y mejora (2).

\section{LA ENSEÑANZA CENTRADA EN EL RESIDENTE Y SU POSIBLE PARALELISMO CON EL CAMBIO EN LAS RELACIONES SANITARIAS}

Una de las razones del nacimiento de la Bioética ha sido la multiplicación de conflictos generados por el cambio en la relación médico-paciente, pasando de una relación paternalista a una consideración del paciente como adulto responsable y capaz de tomar las decisiones sobre las cuestiones de salud de las que él es el principal protagonista. La necesidad de mejorar la comunicación y transmisión de información por parte de los médicos, asignatura aún pendiente en nuestra formación, la mejora con ello del proceso de consentimiento informado, la necesidad de que los profesionales aceptemos que las opciones de diagnóstico y tratamiento ideales para nosotros pueden no serlo para el paciente, la renuncia a potenciales tratamientos que les proponemos aún cuando la renuncia ponga en riesgo su vida, etc., son algunas de las consecuencias de este cambio de relación.

En el proceso enseñanza-aprendizaje también se está produciendo un cambio en su desarrollo clásico. Estamos en un proceso de modificación paulatino que, tratando de preservar lo que ha sido importante y sigue siendo válido del modelo clásico, vaya introduciendo el concepto de enseñanza centrada en el que aprende, o en nuestro caso enseñanza centrada en el residente, y un aumento del campo de la reflexión sobre la práctica (3). Vemos en las tablas II y III las principales modificaciones que supone el cambio de un modelo pedagógico a otro.

El modelo de enseñanza que ha centrado nuestro sistema MIR desde su inicio ha sido el aprendizaje "viendo hacer" e iniciando el hacer (práctica clínica) bajo tutela. Esta fórmula, demostrada válida durante muchos años, permite un más fácil acercamiento al modelo de enseñanza centrada en el alumno. Es así puesto que ya en su origen no estaba centrada en el paradigma clásico, del que sí ha sido un ejemplo la enseñanza durante la licenciatura. En el sistema MIR ha sido esencial el aprendizaje por el modelo, por el ejemplo de otros profesionales, de los especialistas con los que el residente desarrolla su 


\section{TABLA II}

PARADIGMAS DEL APRENDIZAJE

Antiguo

- Interesa qué deberías saber

- Incertidumbre desalienta y la ignorancia se elude

- Aprendizaje por humillación, vergüenza y culpa

- Aprender de expertos reconocidos

- Cantidad de conocimiento asimilable es limitada

- Poderosa intuición

- Dominado por conocimiento procedente de experiencia

- Aprendizaje basado en hechos

- Profesionales en la cima

\section{Nuevo}

- Interesa lo que no sabes y como aprenderlo

- Legitimación de la incertidumbre

- Aprendizaje por cuestionamiento. Transformar problemas en preguntas

- Capaz de cuestionar expertos

- Buscar, encontrar, evaluar y aplicar lo aprendido por experiencia e investigación

- Complementar con el conocimiento adquirido por investigación

- Basado en problemas y procesos

- Profesionales a mano

\section{TABLA III}

ENSEÑANZA CENTRADA EN EL DOCENTE FRENTE A APRENDIZAJE CENTRADO EN EL ALUMNO

Modelo centrado en el tutor

El tutor decide, dirige y controla el proceso de aprendizaje - Relación confianza

- Hace al residente depender de él

- Tutor se responsabiliza de:

- Identificar objetivos del aprendizaje

- Garantizar el aprendizaje

- Evaluar

- Planificar nuevos pasos

- Limita la iniciativa del residente

\section{Modelo centrado en el residente}

El residente es responsable del aprendizaje y como conseguirlo

- Relación confianza

- Tutor comunica al residente su autonomía

- Estimula y apoya al residente para que se responsabilice de:

- Identificar necesidades y objetivos de aprendizaje

- Planificar su aprendizaje

- Realizar el aprendizaje

- Evaluar

- Planificar nuevos pasos

- Facilita el hábito de autoaprendizaje (esencial para la posterior formación continuada)

- Importante papel de la reflexión sobre la práctica realizada formación $(2,4)$. Sin embargo aún así el método clásico del sistema MIR tiene todavía un gran margen de mejora al aplicar el concepto de enseñanza centrada en el residente. Este potencial de mejora viene de la mano de un buen sistema de evaluación formativa, claramente deficitario en nuestro sistema de formación especializada.

El sistema de enseñanza-aprendizaje del MIR debe partir de un buen conocimiento de las características del aprendizaje en el adulto (Tabla IV) (3).

\section{TABLA IV}

\section{CARACTERÍSTICAS DEL APRENDIZAJE EN EL ADULTO}

Los adultos tienden a aprender lo que:

- Es relevante. Los contenidos se adecuan a los fines y los alumnos comparten la importancia de lo que tienen que aprender.

- Se basa y relaciona con sus experiencias previas.

- Está enfocado a problemas concretos. Las situaciones de aprendizaje se parecen a la práctica profesional.

- Es participativo y activo. La teoría se relaciona directamente con los problemas prácticos.

- Es útil en la práctica de inmediato.

- Involucra ciclos de acción y reflexión.

- Se basa en confianza y respeto mutuo. Los alumnos reciben frecuente información sobre su propio aprendizaje (feed-back).

Uno de los cambios principales en el método afecta al tipo de relación entre docentes y alumnos. Frente a la enseñanza por humillación, frente a la potenciación del desequilibrio en la relación, el docente médico debe tratar a los estudiantes y residentes como le gustaría que ellos trataran a los pacientes (5). Esta relación tutor-residente debe ser tal que permita al residente reconocer su incompetencia y no aceptar realizar prácticas para las que no esté preparado o que le originen situaciones de incomodidad moral $(5,6)$. Esta afirmación podría ser más cuestionable con el paradigma clásico de relación paternalista médico-paciente pero no si la aplicamos a la relación hacia la que debemos caminar mediada por un consentimiento informado adulto. En definitiva, qué mejor forma de empezar a enseñar cómo tratar a los pacientes que haciendo vivir a los residentes una relación madura con los docentes, con un papel activo en su aprendizaje, con márgenes para la duda, aprendiendo del error como luego veremos, admitiendo la incertidumbre y no pretendiendo colocar en un falso pedestal al docente.

\section{PROCESO ENSEÑANZA-APRENDIZAJE Y PRINCIPIOS DE BIOÉTICA}

Vamos a ir recorriendo aquí de la mano de los principios de la Bioética una serie de orientaciones como punto de partida para una discusión que mejore la detección, solución y prevención de conflictos éticos ligados a la docencia de la medicina. La ética de "mínimos" como tutores de residentes quedaría conformada por los principios de no-maleficencia y 
de justicia y la de "máximos" por el binomio autonomía-beneficencia.

\section{No-maleficencia}

Cuando hablamos de investigación estamos hablando de una responsabilidad que la sociedad delega en los científicos, asumiendo en el caso de la investigación clínica que una parte de esa sociedad se someterá a un riesgo para generar conocimientos que benefician a toda la sociedad. La sociedad a cambio pide a los investigadores que garanticemos la calidad de dichos proyectos en cuanto a diseños que tengan mayor probabilidad de generar conocimientos generalizables, con el mínimo riesgo posible y garantizando los mecanismos para que los potencialmente sometidos al riesgo no procedan de un proceso de discriminación al ser seleccionados para la investigación.

También la sociedad tiene que asumir que es necesario que los médicos en formación adquieran experiencia para poder beneficiarse luego de ello. En este sentido, como luego veremos, es bueno que haya la mayor información a la población sobre la presencia en los centros sanitarios de profesionales en formación participando en su atención sanitaria, para poder considerar válidos consentimientos implícitos para la realización de estas acciones formativas. En el artículo 104, apartado 1, de la Ley General de Sanidad de 1986 ya decía: "Toda la estructura asistencial del sistema sanitario debe estar en disposición de ser utilizada para la docencia pre, postgraduada y continuada de los profesionales".

Pero por su parte la sociedad debe exigir a la organización sanitaria y a los responsables de este aprendizaje que velemos por la minimización de riesgos. Aquí ésta la principal manifestación del principio de no-maleficencia aplicado a la acción tutorial. El tutor debe proteger al paciente mediante una adecuada supervisión, una adecuada aplicación de la responsabilidad progresiva recogida en la Guía de Formación de Especialistas del Consejo Nacional de Especialidades. Un residente debería empezar a realizar un procedimiento tras conocer la teoría, haberlo visto practicar y, cuando sea posible, haber realizado prácticas de simulación de su aplicación, y haber realizado previamente procedimientos que le preceden en complejidad y adiestramiento (por ejemplo, antes de colocar un catéter pleural haber hecho varias toracocentesis) (Tabla V). La pirámide de Miller diseñada para valorar la capacitación clínica puede servir de guía en este escalonamiento para minimizar los riesgos (7).

Por tanto todo trabajo que en los diferentes programas de especialidades profundice en una mejor definición de estos escalones de responsabilidad progresiva en la formación, siempre entendiendo que los márgenes deben ser amplios, estará poniendo mejores cimientos al principio de no-maleficencia en el proceso de especialización médica.

Mantener viva la conciencia de no competencia del residente o al menos que se autocuestione sobre su competencia o consulte sobre la misma antes de emprender acciones de mayor complejidad, será otro mecanismo de proteger este principio.

La protección de los datos y la intimidad del paciente que sea protagonista de la acción docente, es decir la potenciación de la confidencialidad, forma parte también de este principio.

\section{TABLA V}

SECUENCIA RECOMENDABLE DE MINIMIZACIÓN (NOMALEFICENCIA) EN LOS PROCESOS DE APRENDIZAJE

1. Adecuado aprendizaje de las bases teóricas y secuencia del procedimiento a realizar (conocimiento)

2. Ver realizar el procedimiento a personas expertas (ver hacer)

3. Realizar el procedimiento en condiciones experimentales (maniquíes, simuladores...) (simulación)

4. Haber realizado procedimientos que le precedan en complejidad

5. Práctica clínica tutelada

6. Práctica clínica autónoma

En general asegurar una formación en Bioética del residente potenciará este principio en la acción docente (8). Aunque las normas pueden transgredirse a pesar de conocerlas, un primer motivo de no respetarlas es su desconocimiento, como ponía en evidencia un trabajo realizado con residentes americanos (9).

Este principio llega a su culminación en la responsabilidad que los tutores y los Comités de Evaluación tienen al validar la capacitación como especialista de los residentes a los largo $\mathrm{y}$, principalmente, al final de su período formativo. Volveremos sobre ello al hablar de la evaluación.

\section{Justicia}

Referido a la docencia este principio también puede enunciarse como "tener comportamientos similares ante casos similares y dar respuestas diferentes a casos distintos". En el caso de las acciones de los residentes esto significa que el que el residente realice directamente la atención a un determinado paciente, o una acción concreta de esa atención cómo puede ser una prueba diagnóstica, estará determinado por el nivel de formación en que se encuentre y por el grado de complejidad del problema del paciente. El cumplimiento de esta máxima permitiría dar razones a un paciente de por qué va a ser atendido por un residente (su problema es acorde con el nivel de formación) y por qué el paciente siguiente o el de la cama de al lado no. La mejor delimitación de lo que los residentes tienen que ser capaces de hacer en cada fase de su período especialización facilitará estas decisiones (10).

Este principio encierra también la no discriminación a los pacientes en cuanto a la posibilidad de ser atendidos por médicos residentes.

Un mejor conocimiento público de que como sociedad hemos elegido este sistema de especialización como el mejor, y el más seguro, para dar continuidad a una asistencia sanitaria pública de calidad, hará entender mejor que los riesgos e incomodidades inherentes a este sistema tienen que ser repartidos entre todos. El que esta información conste en los documentos informativos que se entregan a los pacientes al acudir a un centro sanitario, el presentar a los residentes como parte del equipo al llegar un paciente a la planta o a la consulta, etc., contribuirá a reducir problemas. 
Si se hace adecuadamente algunos pacientes pueden vivir como beneficio, e incluso beneficiarse realmente, su participación en el aprendizaje: implica una mayor atención a su caso, el que le atienda más de una persona, el que se comente su caso, etc. (6).

\section{Principios de autonomía y beneficencia}

Los abordamos de forma conjunta por su interrelación dentro del nivel que conocemos como "ética de máximos".

De forma igual o más importante que en el aprendizaje de la parte técnica de la especialidad, en el aprendizaje del respeto a la autonomía de los pacientes, aplicando el principio de beneficencia, es esencial el "modelo o ejemplo", el "ver hacer". Es decir, como tutores o responsables de formación de un residente tenemos la obligación moral de extremar nuestro respeto a la autonomía dentro del proceso de consentimiento informado, como mejor medio de que los residentes lo interioricen. La formación aquí también será gradual, con adquisición progresiva de responsabilidades (11).

Este aprendizaje según el modelo podemos trasladarlo también a la relación entre el docente y al residente. El aprendizaje dentro de un modelo centrado en el alumno supone tratar al residente como un adulto, valorando y potenciando su autonomía y evitando el modelo de enseñanza anterior que incluía poner de manifiesto la ignorancia del alumno, llegando hasta la humillación. Esta nueva estrategia pedagógica favorecerá que el residente aprenda un modelo de relación con el paciente potenciador de la autonomía (5).

La aceptación de las individualidades y las sugerencias del residente queda enmarcada en este principio. El no abandono, el apoyo personal ante los problemas surgidos durante la residencia, etc., servirán para mostrarle el modelo de comportamiento beneficente con el paciente. Un trabajo que recoge los motivos por los que residentes americanos reconocían haber transgredido las guías éticas indica que uno de ellos es el haber visto que así lo hacían sus docentes (9). Es un dato que evidencia la importancia del aprendizaje mediante el ejemplo. Trabajando junto a los residentes y estudiantes tenemos, queramos o no, un papel como modelos a seguir.

Veremos también a continuación el posible conflicto entre la autonomía de los pacientes y la formación de residentes.

\section{CONSENTIMIENTO INFORMADO Y FORMACIÓN DE RESIDENTES}

Además de lo ya comentado al hablar de los principios de autonomía y beneficencia, quisiera en este espacio buscar respuestas a la pregunta de si los pacientes tienen que conocer que la persona que les realiza una técnica, una atención sanitaria, está en periodo de formación. ¿Es necesario el consentimiento informado para los procedimientos aprendizaje que implican a pacientes? Por supuesto que cuando me refiero a consentimiento informado me estoy refiriendo al proceso y a la información verbal y no al hecho de que haya que elaborar documentos especiales de consentimiento informado. Vamos a recorrer algunas situaciones "tipo", algunos ejemplos (sabiendo que la introducción de múltiples matices puede hacer discutibles y variables los ejemplos), que nos puedan facilitar después sistematizar la respuesta a esta pregunta.

A) Prácticas en cadáver para el aprendizaje. Empecemos por aquí, por ir de lo más sencillo a lo más complejo. Aunque también es un tema que admite el debate, hay autores que han argumentado que no es preciso el consentimiento informado siempre que se limiten a procedimientos que no mutilen el cadáver, que sean debidamente tuteladas y restringidas a personas que se espera que se desenvuelvan en el área de conocimiento de las prácticas que están realizando (12). Por tanto el primer paso también aquí es asegurar la no-maleficencia. De todas maneras lo adecuado para considerarlo así sería que la sociedad tuviera suficiente información como para que el silencio, la no negación expresa previa por los pacientes, sea interpretado como un consentimiento implícito para la realización de estas prácticas.

B) Un segundo ejemplo puede ser el de un residente de primer año, o incluso un estudiante de medicina, que es enviado a "repetir" la anamnesis y exploración física general de un paciente que ya lleva unos días ingresado.

C) La realización de una primera punción lumbar por un residente de primer año en un paciente ingresado en que dicha punción debe realizarse para un estudio de enfermedad neurológica, en situación no urgente.

D) Un cuarto caso puede ser la primera punción lumbar de un residente de primer año pero en condiciones de urgencia ante la sospecha de una meningitis bacteriana aguda.

E) Un residente realiza su primera intubación orotraqueal en un paciente que va a ser intervenido de forma programada, bajo la dirección del anestesista, o bien un residente va a participar por primera vez de forma activa en una intervención quirúrgica.

F) Realización de una segunda exploración ginecológica por el residente tras la realizada por el adjunto.

G) El adjunto propone al residente la colocación de un catéter de Schwan-Ganz para monitorización hemodinámica a un paciente que no lo requiere estrictamente, para permitir que practique este procedimiento.

Estas oportunidades clínicas de aprendizaje, y creo que la mayoría de las que se nos pueden ir ocurriendo, podemos clasificarlas en dos grandes grupos (Tabla VI):

1. Procedimientos diagnósticos y/o terapéuticos necesarios para el paciente y que además se utilizan como oportunidades para el aprendizaje.

2. Procedimientos sólo realizados para aprendizaje. Entre estos, a su vez, encontramos algunos inocuos, no invasivos o con riesgo mínimo y otros invasivos o que implican riesgo para el paciente.

En este segundo grupo, realizado sólo para el aprendizaje, probablemente es fácil estar de acuerdo que requerirán el consentimiento informado del paciente, incluso aquellos en principio inocuos. Por ejemplo, en el caso B lo adecuado es indicar al paciente que va a ir un médico en formación a repetirle algunas preguntas y la exploración física que nosotros ya le hicimos al ingresar. Todos estos procedimientos, incluso los más sencillos, tienen que estar sometidos antes de planteárselos al paciente a los pasos de minimización de riesgos (e incluso molestias), es decir, de preservación de la no-maleficencia: que el residente conozca (teoría) y haya presenciado (ver hacer) exploraciones físicas, que conozca bien la obligación de confidencialidad, etc. y que en lo posible lo haya practicado sobre maniquíes o simuladores (Tabla V) Probablemente hay algunos con un nivel de riesgo o invasividad tal, como puede ser presupuesto G, que no es aceptable su planteamiento aún con el consentimiento del paciente. 


\section{TABLA VI}

CLASIFICACIÓN DE LOS PROCEDIMIENTOS DE ENSEÑANZA

EN LA CLÍNICA DE CARA A LA MEJOR VALORACIÓN DE POTENCIALES CONFLICTOS ÉTICOS

1. Procedimientos diagnósticos y/o terapéuticos necesarios y que además se utilizan para el aprendizaje

\section{Sólo realizados para el aprendizaje - Inocuos, no invasivos, bajo riesgo$$
\text { -Invasivos }
$$

En cualquier caso tanto en el primer como en el segundo grupo hay que tratar que el paciente se sienta un docente del residente y no un simple medio de aprendizaje $(6,13)$.

Respecto al primer grupo, procedimientos necesarios al paciente, voy a presentar antes algunos datos más que nos ayuden a la deliberación.

\section{LA ENSEÑANZA DE LOS “ERRORES” Y EL "NO LO SÉ" COMO MOTORES DEL APRENDIZAJE}

Un ejemplar de 1999 del British Medical Journal (14), dedicado a la necesidad de introducir procedimientos para sustentar con pruebas los métodos de enseñanza-aprendizaje, se iniciaba con el sugerente título de: "Las tres palabras más importantes en la educación (I don't know - No lo sé)". El profesor Pencheon, docente e investigador de Cambridge, insistía en que actualmente lo más importante que tenemos que saber es "qué es lo que no sabemos". Aprender consiste en darse cuenta de lo que no sabes. Esto destruye el paradigma clásico de la enseñanza centrada en el docente en que la falta de certeza desestabiliza al docente y la ignorancia estaba prohibida en su ámbito (Tabla II).

Como dice uno de los principios de Karl Popper para una nueva ética científica: "Es imposible evitar todos los errores e incluso todos aquellos que, en sí mismos, son evitables. Todos los científicos cometen equivocaciones continuamente. Hay que revisar la antigua idea de que se pueden evitar los errores ya que, por lo tanto, en sí misma encierra un error".

Por un lado este recuerdo quiere estimular la importancia que tiene el aprendizaje a partir de los errores en nuestra actividad cotidiana. Pero, por otro lado, traigo aquí el tema porque creo que el aumento del conocimiento por parte de la sociedad, de todos los pacientes potenciales, de que la medicina es una ciencia no exacta sino probabilística y que puede no haber resultados aunque se hagan bien los procesos y que las desviaciones de lo esperable son posibles, también facilitará la aceptación de lo que estamos tratando. Es decir, facilitará la aceptación de la participación de profesionales en periodo de formación en nuestra atención sanitaria. Las desviaciones de lo esperado, muchas veces vividas por los pacientes como "errores", son inherentes al trabajo también de los más experimentados o menos novatos. Los ciudadanos deben saber que la medicina es una profesión obligada a poner unos medios, los adecuados a la situación clínica en ese momento preciso, pero no a obtener unos resultados. Pero además, dada la diversidad de situaciones clínicas, de pacientes y de contextos sani- tarios, es preciso establecer por el facultativo la aplicación de decisiones prudentes y razonables de los criterios genéricos a situaciones concretas.

Por otro lado, como tutores nuestra obligación es mantener al residente, sobretodo a aquel más inconsciente, consciente de su nivel de incompetencia o competencia parcial.

En este contexto es importante la máxima de "ser tolerantes con el error técnico e intolerantes con el error moral" (5).

ALGUNAS VÍAS DE RESPUESTA DESDE LA ÉTICA DE MÍNIMOS (LEGISLACIÓN) Y LOS CÓDIGOS ÉTICOS PROFESIONALES

-Códigos éticos profesionales.

Empezando por los códigos éticos profesionales encontramos que el código deontológico de la OMC no se detiene sobre estas cuestiones. En el código ético americano (15) encontramos al menos dos referencias a las cuestiones que nos ocupan:

- El médico tiene la responsabilidad de enseñar la ciencia, el arte y la ética de la medicina a estudiantes, residentes y otros médicos, así como de supervisar a aquellos a los que está enseñando.

- Debe informarse a los pacientes de la condición de médicos en capacitación si intervienen en su atención.

- La Ley General de Sanidad de 1986.

Aunque algunos artículos de ley han sido derogados por la Ley Básica de Autonomía de los pacientes, a la que luego nos referiremos, entiendo que hay otros que influyendo en el tema que nos ocupa, permanecen vigentes:

- Artículo 10 (ap. 4): "Todos tienen derecho a ser advertidos de si los procedimientos pueden ser utilizados en función de un proyecto docente o de investigación...".

- Artículo 104 (ap. 1): “ Toda la estructura asistencial del sistema sanitario debe estar en disposición de ser utilizada para la docencia pregraduada, postgraduada y continuada de los profesionales".

- Ley Básica de Autonomía de los pacientes y de la Historia Clínica (Ley 41/2002).

- Art. 4(ap. 1): Los pacientes tienen derecho a conocer, con motivo de cualquier actuación en el ámbito de su salud, toda la información disponible sobre la misma, salvando los supuestos exceptuados por la Ley... La información, que como regla general se proporcionará verbalmente dejando constancia en la historia clínica, comprende como mínimo, la finalidad y la naturaleza de cada intervención, sus riesgos y sus consecuencias.

- Art. 5 (ap. 4): "El derecho a la información puede limitarse por la existencia acreditada de un estado de necesidad terapéutica" (es decir, que su conocimiento pueda perjudicar su salud de manera grave) (clásicamente denominado "privilegio terapéutico").

- Art. 8 (ap. 4): Reitera lo indicado en la LGS de 1986: "Todo paciente o usuario tiene derecho a ser advertido sobre la posibilidad de utilizar los procedimientos de pronóstico, diagnóstico y terapéuticos que se le apliquen en un proyecto docente o de investigación, que en ningún caso podrá comportar riesgo adicional para su salud".

- Artículo 10. Condiciones de la información y consentimiento por escrito.

1. El facultativo proporcionará al paciente, antes de recabar su consentimiento escrito, la información básica siguiente: 
- Las consecuencias relevantes o de importancia que la intervención origina con seguridad.

- Los riesgos relacionados con las circunstancias personales o profesionales del paciente.

- Los riesgos probables en condiciones normales, conforme a la experiencia y al estado de la ciencia o directamente relacionados con el tipo de intervención.

- Las contraindicaciones.

2. El médico responsable deberá ponderar en cada caso que cuanto más dudoso sea el resultado de una intervención más necesario resulta el previo consentimiento por escrito del paciente.

- Artículo 16 (ap. 3): “...El acceso a la historia clínica con fines judiciales, epidemiológicos, de salud pública, de investigación o de docencia se rige por lo dispuesto en la Ley Orgánica 15/1999 de Protección de Datos de carácter personal, y en la Ley 14/1986 General de Sanidad. Obliga a preservar los datos de identificación, separados de los clínico-asistenciales, de manera que como regla general quede asegurado el anonimato, salvo que el paciente haya dado su consentimiento para no separarlos".

\section{LA EVALUACIÓN FORMATIVA COMO PROCESO MORAL Y COMO PRINCIPAL POTENCIAL DE MEJORA DEL SISTEMA MIR}

Como dicen Prados y Santos en su artículo sobre evaluación de los MIR como proceso de aprendizaje (16), la evaluación además de ser un proceso técnico es un proceso moral. La ética del proceso evaluativo condiciona sus aspectos técnicos y la técnica de su desarrollo tiene implicaciones morales. La evaluación no es un fenómeno aséptico, que se puede realizar sin preguntarse por los valores, por el respeto a las personas, por el sentido de la justicia. Importa mucho saber a qué valores sirve y a quienes beneficia. Aunque la subjetividad (que no debe confundirse con arbitrariedad) no podrá desaparecer completamente de este proceso puede ser minimizada con las mejoras.

La mejora en el proceso de evaluación, tratando de caminar hacia la excelencia de una evaluación centrada en la progresión en el aprendizaje, es el principal reto actual de nuestro sistema MIR. Está además ligado al otro gran reto que es el reconocimiento y potenciación del papel de los tutores, especialmente, y del resto de participantes en las acciones docentes.

El hecho tiene completa vinculación con el tema que nos ocupa porque la mejora en un proceso de evaluación así entendido y que contempla el respeto a los valores de los implicados, permitirá mejorar y garantizar la protección de los otros agentes implicados en las acciones docentes, los pacientes. Un buen proceso de evaluación formativa garantiza la minimización de riesgos en el proceso docente y además potenciará la reflexión sobre los temas que aquí debatimos.

Pero además la evaluación tiene un contenido social ya que trata de garantizar que los profesionales dominan los conocimientos, tienen las habilidades y disponen de las actitudes que permiten asegurar que el ejercicio de su práctica profesional será bien realizado (16).

Por otro lado hay que recordar aquí que esto no queda limitado a la fase de especialización, sino que el título de especialista no concede por sí de modo indefinido la necesaria competencia (entendiendo por tal buen conocimiento y ade- cuada destreza). Esta se debe mantener mediante los programas de formación continuada. Todo médico debe conocer los límites de su competencia.

\section{ALGUNAS POSIBLES RESPUESTAS TRAS ESTE CAMINO RECORRIDO}

En el apartado 4 dejé abierta la respuesta a si era necesario el consentimiento informado para las acciones que realizan residentes en el segundo grupo de acciones que habíamos delimitado (aquellas que siendo necesarias para el abordaje del problema asistencial del paciente se utilizan además como medios de aprendizaje). Después de lo revisado enuncio aquí algunas posibles vías de respuesta que permitan proseguir el debate, puesto que como se verá algunas son compatibles entre sí pero otras son excluyentes:

- $\mathrm{Si}$ un residente va a realizar un procedimiento que corresponde a su nivel de formación (por ejemplo atención en urgencias a patología de "medicina general") aunque sea con menos experiencia que otros médicos (este hecho siempre se dará sea quien sea el médico que le atienda), no habría por qué indicar explícitamente que es un residente el que lo hace. Por lo mismo no es sostenible decir que un residente de primer año no puede firmar informes de alta de urgencias (esto diferencia también la respuesta a varios temas tratados aquí respecto a estas mismas situaciones planteadas en estudiantes de medicina, puesto que residente ya ha alcanzado una capacitación básica según los mecanismos admitidos actualmente por nuestra sociedad). La respuesta será distinta cuando son las primeras veces que se hace un procedimiento de "especialista".

—En el trabajo actual en equipo, los riesgos "mínimos" (si se cumplen los procedimientos progresivos de aprendizaje) de la realización de técnicas por los residentes deben considerarse incluidos en la media de riesgos inherente a la práctica de ese procedimiento en cada centro concreto. Esta media es la que se debería transmitir a los pacientes a la hora de plantear les la información necesaria para solicitar su consentimiento. La potencialidad de riesgos de alguna manera también tiene un rango de variabilidad entre las distintas personas ya especialistas que realizan el procedimiento: una cosa es la capacitación y otra la experiencia, siendo el margen de variabilidad de ésta muy amplio entre los especialistas de nuestro sistema sanitario. Todo proceso de consentimiento informado debería terminar con una pregunta abierta: ¿desea usted saber algo más? En caso de que la persona demande explícitamente esta información será el momento de proporcionarla.

-En los procedimientos en que se decida que es conveniente informar de que va a intervenir un residente para solicitar consentimiento, lo habitual es que este proceso sea verbal, siendo la excepción el que haya que realizar un registro escrito de esta parte del consentimiento.

- Sin pretender la defensa de una vuelta a la generalización paternalista del denominado "privilegio terapéutico", sí hay que ser conscientes de que estas informaciones en algunos momentos sólo añaden tensión y preocupación a personas ya suficientemente inquietas por su propio proceso de enfermedad y por el procedimiento a que van a ser sometidas. Será perfectamente invocable esta limitación al derecho a la información, que como hemos dicho recoge la Ley Básica en su apartado 5 como "existencia de un estado de necesidad terapéutica". 
ALGUNOS PROBLEMAS ÉTICOS RELACIONADOS CON LOS AVANCES TECNOLÓGICOS Y CON LA GENERALIZACIÓN DE LA ASISTENCIA SANITARIA

Ya he dicho que estas son dos de las causas de aumento de problemas éticos en la práctica clínica. También estas dos raíces nos pueden servir de guía para suscitar el debate sobre algunos problemas relacionados con la docencia especializada.

${ }_{-}^{-}$iY si un paciente se niega a ser tratado por un residente? El logro del derecho a la asistencia sanitaria por todos los ciudadanos ha generado problemas en cuanto a la justa distribución de los recursos sanitarios, si no escasos al menos sí limitados, ante la demanda creciente de "salud". Esa atención se desarrolla en centros públicos o privados concertados, muchos de los cuales son centros docentes para especialización médica. Los pacientes en general son colaboradores y aceptan, de manera implícita y también explícita cuando se lleva a cabo este consentimiento, su participación en este sistema elegido por nuestra sociedad para formar a los especialistas. En caso de que esto no sea así, ¿tienen derecho a que se acepte su negativa a la participación de un residente en su asistencia?

En caso de producirse, como todo conflicto ético, debe abordarse con método y prudencia. Lo primero sería intentar averiguar las causas de esa negativa. Una falta de conocimiento de lo que es el residente, lo que es el sistema de formación MIR, las garantías y supervisión que lleva inherente, etc., puede estar en el origen de ese rechazo. Se debe garantizar que el procedimiento en que va a participar en residente es acorde con su nivel de formación: que está capacitado para ello, aunque tenga una limitada experiencia. Incluso es bueno que los pacientes sepan que de esa atención pueden verse especialmente beneficiados a veces: la intervención de personas en aprendizaje muchas veces implica que esos casos sean especialmente o doblemente revisados, con búsqueda de información actualizada basada en la evidencia, presentados a otros médicos, etc. (6). Recordemos que este beneficio puede no ser siempre así pero lo que sí es generalizable es que la presencia de residentes en un servicio obliga a un mejor cumplimiento de las obligaciones de formación continuada de los especialistas del servicio, lo cual redunda en beneficio de los pacientes.

Si persiste la negativa en casos de procedimientos que sólo se hacen por motivos de aprendizaje (por ejemplo, una segunda exploración física) hay que permitir un ambiente de especial tolerancia para la persistencia de esta negativa.

Sin embargo, aunque sé que es discutible, en caso de tratarse de procedimientos del segundo tipo (necesario para la asistencia al paciente) creo que es moralmente admisible insistir en que conozca bien que su negativa vulnera de alguna manera el principio de justicia, en cuanto a la obligación de repartir entre todos esta "carga" que acompaña a la distribución justa de los recursos sanitarios. Al ser un principio de nivel de ética de mínimos, para incumplirlo habría que justificarlo como una excepción, basándonos en las consecuencias o por vulnerar otro principio del mismo rango (no-maleficencia). En este caso se admitiría por el potencial riesgo para la salud del paciente generado por la tensión de la relación médico-paciente si persistiéramos en que la atención fuera realizada por el residente.

-Tutela de los residentes en urgencias. Una de las consecuencias del gran logro social que ha sido el derecho generalizado a la asistencia sanitaria es la masificación de algunos ser- vicios y las consecuentes listas de espera. Uno de los servicios más afectados son los de urgencias. En un encuentro de personas dedicadas a la docencia especializada celebrado en 2004, dentro del proceso de constitución de la Asociación de Redes Docentes y Asesoras, bajo el título de "Profesionalización del tutor de postgrado", se ponía en evidencia la insuficiencia de la tutela de residentes en urgencias como un problema generalizado $(17,18)$. Se hicieron una serie de recomendaciones, entre las cuales destaca una llamada a que durante las primeras fases de la formación debía existir una tutela directa (ya que se denunciaba incluso la existencia, a veces, de tutelas "localizadas", a distancia, en estas primeras fases en algunas especialidades). Sin pretender con esto dejar de admitir que los residentes ya en urgencias tienen que responsabilizarse precozmente de acciones de "medicina general", como ya ha quedado referido anteriormente, sí hay que indicar que la ausencia de tutela adecuada es no sólo un problema técnico sino también ético, en el ámbito de la no-maleficencia. Podría en algún momento justificarse, siempre como excepción, por una sobrecarga no esperable del servicio (justificación para preservar el principio de justicia, para atender a todos los pacientes en ese momento puntual), pero no convertirse en un hecho generalizado y reiterado, como lamentablemente ocurre.

- Desarrollo tecnológico de simuladores para la docencia. Los avances técnicos han permitido el desarrollo de materiales y modelos para el aprendizaje de técnicas invasivas que reproducen cada vez mejor las relaciones anatómicas, sensaciones, cambios de características tisulares, etc., que podemos encontrar tanto en personas sanas como en pacientes con patologías definidas.

La falta de disponibilidad de estos materiales hace que del escalón de "ver hacer" una técnica se salte a la realización de la misma por primera vez en un paciente, o en su caso en una persona voluntaria. La disponibilidad progresiva de material de simulación va haciendo que haya que ir incluyendo, siempre progresivamente y dependiendo de la disponibilidad de recursos, dentro de las obligaciones de no-maleficencia de la acción tutorial la realización de prácticas en simuladores antes de pasar a la práctica clínica con pacientes.

Es verdad que muchos de estos materiales de simulación son todavía inaccesibles económicamente para muchos centros docentes. Aparece aquí otro de los problemas que tiene la formación especializada: se ha asumido que la formación se podría incorporar a los centros asistenciales sin más medios que los salarios de los residentes y la utilización de los médicos que realizan asistencia como modelos para la enseñanza mediante el ejemplo. Una docencia MIR de calidad requiere recursos materiales. Hemos argumentado ya una parte importante de ellos, que son los que hay que dedicar a mejorar la formación pedagógica, la formación en métodos de enseñanza-aprendizaje y de evaluación de los docentes. Aquí aparece un segundo apartado que justifica el aumento de medios materiales: la necesidad de progresar en el aprendizaje mediante simuladores antes de llegar a la práctica clínica. Hay una forma de poner en marcha una mejora en este escalón docente y es la creación de una base de materiales de simulación financiados por la Comunidad Autónoma o por cada cierto número de centros docentes, que centralice el material de simulación básico para que sea utilizado de forma secuencial, en forma de préstamo, para acciones docentes concretas, por los distintos centros docentes adscritos. 
Por tanto la mejora científico-técnica también debe beneficiar desde este punto de vista a los pacientes.

La dedicación de recursos a estos dos aspectos, formación de docentes y disponibilidad de materiales de simulación, no sólo suponen beneficio en la formación técnica de nuestro residentes sino en la reducción de algunos de los problemas éticos en que nos estamos centrando.

- Confidencialidad de los datos sanitarios. La protección de la intimidad de los pacientes, incluyendo en ella la protección de sus datos sanitarios, es fuente de problemas éticos en la asistencia y en la investigación. Aunque es mencionado este tema aquí por la frecuente afirmación de que los avances tecnológicos han aumentado el riesgo de vulneración del derecho a la intimidad (informatización de datos sanitarios) o de sus consecuencias (información genética) es un problema anterior y que va más allá de esta afirmación. No se puede resolver sólo con medidas de obstaculización física del acceso a los datos si no hay acciones formativas adecuadas (19). Algún estudio ha puesto en evidencia las dificultades de comprensión adecuada, o al menos de adecuación a la práctica, de acciones formativas teóricas sobre la protección de este derecho, en alumnos de medicina (20).

El adiestramiento en la importancia de esta protección es esencial durante la formación médica especializada y una forma especial de educar en este sentido es la protección de estos datos cuando se utilizan para acciones docentes. Es obligación de los formadores cuidar que se eviten las fuentes de identificación de pacientes en sesiones docentes, en informes de recopilación de datos en memorias docentes, etc.

- Conflictos de intereses y relaciones con la industria farmacéutica. Siguiendo con la importancia del aprendizaje a través del modelo (4), del ejemplo, será importante que los residentes perciban claridad respecto a potenciales conflictos de interés en el patrocinio de acciones docentes y en las ayudas aportadas a través de la empresa privada para acciones docentes.

En las acciones relacionadas con investigación, la asistencia y la formación continuada hay que ser conscientes de estos potenciales conflictos interés y una forma de saber hasta qué punto pueden ser tolerables es preguntarnos cómo serían valorados estos conflictos por nuestros pacientes y por la sociedad si los conocieran. En el campo de la docencia habría que incluir además la interrogación sobre qué van a pensar los residentes o cómo va a influir en su aprendizaje.

\section{PROPUESTAS Y CONCLUSIONES}

Aunque en el día a día en la clínica las oportunidades docentes surgen y hay que aprovecharlas, y ese ha sido uno de los éxitos del sistema MIR, tanto desde el punto de vista técnico como ético son previsibles muchas de las características de dichas oportunidades. Aunque no podamos programarlas siempre en cuanto al momento concreto en que se van a presentar, sí puede programarse cómo enfrentarnos a ellas cuando surjan y cómo hacer que el paciente se sienta mejor dentro de ellas (13).
Hacer conscientes, enunciar y ayudar a hablar de problemas que existen o de potenciales fuentes de problemas no es hacer más compleja la práctica, en este caso la docente, sino que sólo es poner en evidencia la complejidad ya existente. Definir, dar nombre, clasificar los problemas es el inicio del camino para solucionarlos. Este ha sido el objetivo de este texto. Resumo aquí algunas vías de disminución de problemas que el texto ha pretendido aportar:

- Aumentar el conocimiento genérico por parte de los ciudadanos de que existe docencia pre y postgraduada en nuestros centros asistenciales y que se recuerde esta información cuando acudan a nuestros centros.

- Aumentar y asegurar los métodos de minimización de riesgos para los pacientes en el proceso de aprendizaje. Hacer conscientes a la sociedad y organización sanitaria que esto implica aumento de recursos materiales, esencialmente para la formación y reconocimiento de tiempo para los docentes (17) y para incorporación de materiales de simulación a la enseñanza.

- Progresar en el debate y reflexión sobre si es necesario un consentimiento explícito por parte de los pacientes en algunas de las situaciones que hemos reflejado.

- Mejorar la cultura del aprendizaje de los errores y la conciencia de incompetencia o competencia parcial como generadora de prudencia y respeto moral.

- Mejorar la conciencia por parte de la sociedad de que la medicina es una ciencia probabilística, obligada a poner los medios adecuados pero no a garantizar resultados.

- La mejora del proceso de evaluación formativa y "profesionalización" de los tutores contribuirá a disminuir las fuentes potenciales de problemas éticos.

En cualquier caso estoy convencido que suscitar discusión y debate sobre lo que hacemos y como mejorarlo ya es en sí mismo formativo y, en este caso, una herramienta de mejora de la docencia médica.

\section{DEDICATORIA}

"A los pacientes como docentes".

Cuando terminaba este texto, el día de San Andrés de 2005, Andrés fallecía a sus 94 años. Sin entrar en detalles de la excepcionalidad de las manifestaciones clínicas de la patología que vivió en los últimos años de su bien disfrutada vida, simplemente decir que la duración excepcional de su ascitis refractaria (5 años) obligó a plantear paracentesis evacuadoras periódicas, en algunas fases cada 15 días y luego más esporádicamente. Como consecuencia de ello ha sido uno de esos pacientes "maestros" que comento en el texto. Ayudó, ya que era conocedor de este hecho, a que aprendieran a realizar esta técnica varias generaciones de residentes que se han formado en nuestra Área Sanitaria.

Con él dedico este texto a los pacientes que como él, conscientemente o sin conciencia clara de esa contribución, permiten que se formen los especialistas de nuestro sistema sanitario. 


\section{Bibliografía}

1. Stone S, Ellers B, Holmes D, Orgren R, Qualters D, Thompson J. Identifying oneself as a teacher. The perceptions of preceptors. Med Educ 2002; 36: 180-5.

2. Wright SM, Kern DE, Kolodner K, Howard DM, Brancati FL. Attributes of excellent attending-physician role models. New Engl J Med 1998; 339: 1986-93.

3. Kaufman DM. Applying educational theory in practice. BMJ 2003; 326 : 213-6.

4. $\quad$ Bligh J. Learning by example. Med Educ 1999; 33: 234-5.

5. Reiser SJ. The ethics of learning and teaching medicine. Acad Med 1994; 69: 872-6.

6. Coldicott Y, Pope C, Roberts C. The ethics of intimate examinationsteaching tomorrow's doctors. BMJ 2003; 326: 97-101.

7. Miller GE. The assessment of clinical skills/competence/performance. Acad Med 1990; 65(9 Suppl): S63-7.

8. Garrido JA, González-Moraleja J. Una experiencia de formación en Bioética en el Programa Común Complementario de los residentes. Abstracts del V Congreso Nacional de Bioética; 6-8 de Junio de 1999; Madrid; Pag 35.

9. Green MJ, Mitchell G, Stocking CB, Cassel CK, Siegler M. Do actions reported by physicians in training conflict with consensus guidelines on ethics? Arch Intern Med 1996; 156: 298-304.

10. Consejo Nacional de Especialidades Médicas. Guía de formación de especialistas. Centro de Publicaciones, Secretaria General Técnica.
Madrid: Ministerio de Sanidad y Consumo, 1986.

11. Garrido JA. Formación en comunicación y consentimiento informado en nuestro sistema de residencia: ¿qué información puede y debe dar un residente? Med Clin (Barc) 1998; 111: 739-41.

12. Solsona JF, Sucarrats A. Prácticas en cadáver para la enseñanza. ¿Se debería pedir el consentimiento informado? Med Clin (Barc) 2001; 117: 351-2.

13. Spencer J. Learning and teaching in the clinical environment. BMJ 2003; 326: 591-4

14. Editor's choice. "I don't know": the three most important words in education. BMJ 1999; 318: 1223.

15. Snyder L, Leffler C; Ethics and Human Rights Committee, American College of Physicians. Ann Intern Med 2005; 142: 560-82.

16. Prados JD, Santos MA. La evaluación de los MIR como un proceso de aprendizaje. Med Fam (Andalucía) 2000; 1: 78-83.

17. Instituto de Salud Carlos III y Asociación de Redes Docentes y Asesoras. Profesionalización del tutor de postgrado. II Encuentro Lazareto de Mahón. Educación Médica 2004; 7: 105-6.

18. Tutosaus JD. Fundación de AREDA. Educación Médica 2005; 8: 10-13.

19. Garrido JA. Indiscreción y derecho a la intimidad en el sistema sanitario. Med Clin (Barc) 2000; 114: 358-9.

20. Graham H, Clayden G, Greenough A. Medical students and patient confidentiality: how effective is confidentiality training? 11th international Ottawa conference on Medical education; 2004, July 6-8; Barcelona. Educación Médica 2004; 7: 43 